\title{
Time domain model of the resonance-based electromagnetic pulses emission system
}

\author{
Mikołaj Nowak ${ }^{1}$, Kazimierz Jakubiuk ${ }^{1, *}$, Daniel Kowalak ${ }^{1}$, and Mirosław Wołoszyn ${ }^{1}$ \\ ${ }^{1}$ Gdańsk University of Technology, Faculty of Electrical and Control Engineering, Narutowicza Str. 11/12, 80-233 Gdańsk, Poland
}

\begin{abstract}
In the literature, the emission properties of the electromagnetic field pulse systems based on high voltage antennas (HVR) have been widely described. In order to increase the emission efficiency by extending duration and increasing amplitude of the impulse it is possible to use a resonance system tuned to the parameters of the signal shaped in the pulse forming circuit. The synthesis of a time-domain simulation model of a complex electromagnetic pulses emission system and its verification by experimental research has been presented in this paper.
\end{abstract}

\section{Introduction}

An important element of the electromagnetic field generation and emission systems is the antenna powered from a source of high voltage pulses $[1,3,4]$. The classic approach to this type of systems assumes the construction of low-quality factor $\mathrm{Q}$ broadband antennas (UWB) which have lower gain and worse directional properties compared to narrowband designs. In the case where the purpose of the emission system is to efficiently transmit energy without necessity to maintain the shape of a pulse, it is possible to use resonant systems in which one of the resonant elements LC is the capacity or inductance of the antenna. By increasing the value of the system Q-factor and extending the duration of the signal, it is possible to achieve higher radiation efficiency [3]. The concept of a low-frequency (order of $\mathrm{MHz}$ ) resonance pulse system based on the construction of a spiral antenna (Fig. 1) powered by fuse-based high voltage pulse forming circuit [2] has been presented in this paper.

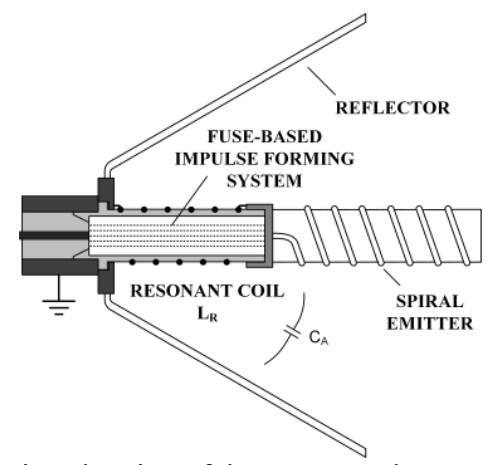

Fig. 1. Overview drawing of the resonant electromagnetic pulse emission system with the capacity $C_{A}$ of the antenna and the inductance $L_{R}$ of the external resonance coil.

Due to the necessity of tuning the resonant branch of the system (by changing the external inductance) to the frequency of self-radiation of the antenna, as well as monitoring the influence of antenna impedance on the process of forming high voltage pulses by the fuse, it is helpful to construct a circuit-based model that predicts the reverse effect of the antenna on the forming system and its radiating effect on the environment.

\section{Model of the emission system}

The appropriate selection of the antenna elements in the time domain can be carried out by means of a natural division into the field-coupling zones of the antenna - the reaction and radiation zone. Assuming that for the considered low frequency range it is possible to overcome phenomena related to the wave reflection in the far field and thus the influence of the radiation environment on the currents and voltages in the antenna is negligible, it is possible to extract the equivalent model parameters directly from the calculated antenna field distribution in the reaction zone. The antenna geometric model used for simulation purposes in the CST Studio simulation software [5] and its actual physical model have been shown in Fig. 2.

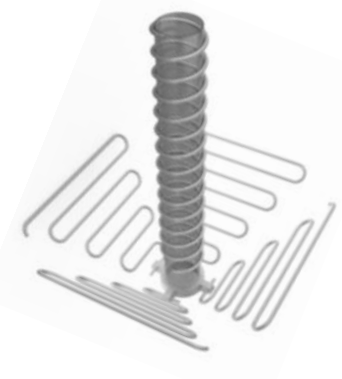

a)

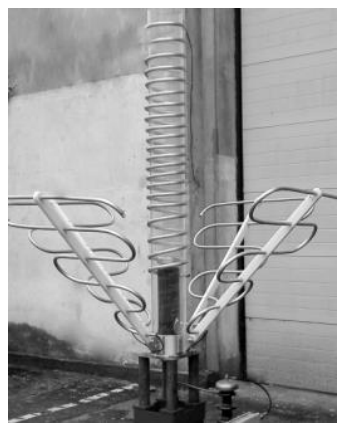

b)
Fig. 2. Geometric (a) and physical (b) model of the resonant electromagnetic pulse emission system.

On the basis of the frequency response of the system impedance the values of the reaction-zone equivalent circuit parameters have been determined [3]. The

\footnotetext{
Corresponding author: kazimierz.jakubiuk@pg.edu.pl
} 
fundamental model for their specification was a modified spiral antenna equivalent model integrated with the electromagnetic field formation and emission system (Fig. 3).

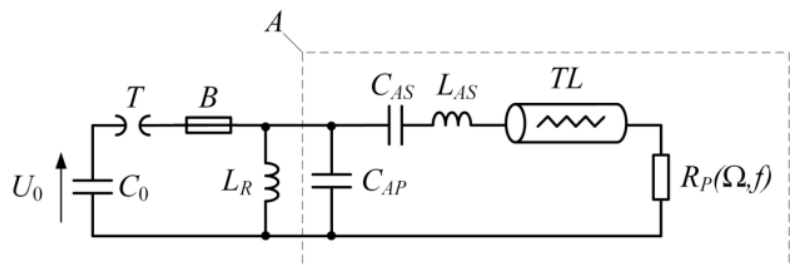

Fig. 3. Dynamic circuit model of the electromagnetic field pulse emission system, $C_{0}$ - capacitor bank, $T$ - trigatron, $B$ - fuse, $L_{R}$ - resonant coil, $A$ - antenna model, $C_{A P}$ - parallel antenna capacity, $C_{A S}$ - serial antenna capacity, $L_{A S}$ - antenna inductance, $T L$ - parametric model of lossy transmission line, $R_{P}(\Omega, f)$ - radiation resistance.

The influence of the antenna in the far field had been modeled using the lossy long line model [3]. Radiation resistance parameter $R_{P}$ of the antenna describing the amount of radiated energy was determined based on field simulations of the antenna and described using a nonlinear model for a specific radiation environment [3].

\section{Experimental verification of the model}

In the measuring system whose schematic diagram has been presented in Fig. 4 a series of measurements was performed to verify the presented time domain model. The values of the dominant component of the electric field $E$ was recorded in the distance from the antenna $x \epsilon<1 m ; 6 m>$.

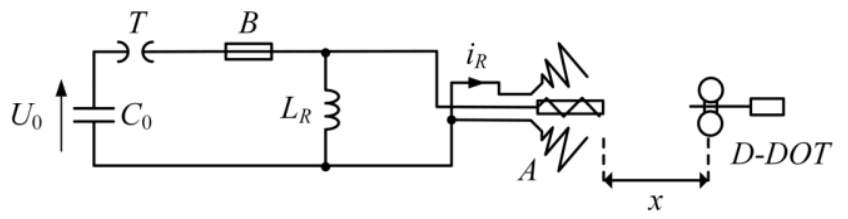

Fig. 4. Schematic diagram of the setup for measuring the electric field $\mathrm{E}$ of the resonance-based pulse emission system.

The measurements results of the electric field $E(t)$ waveforms at a distance of $4 \mathrm{~m}$ from the antenna, compared with the results obtained by simulation method are presented in the Fig.5.

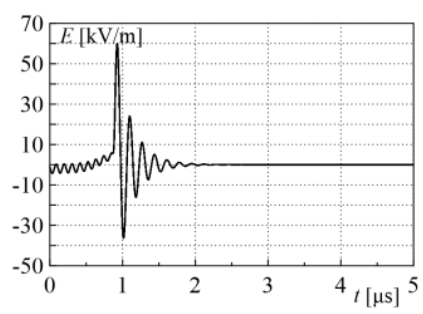

a)

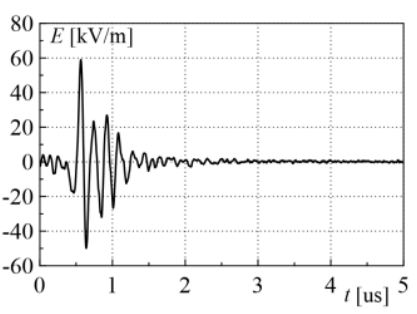

b)
Fig. 5. Waveforms of the dominant component of the electric field $E(t)$ at a distance of $4 \mathrm{~m}$ from the antenna obtained by a) simulation and $b$ ) experimental research.

Based on the simulation of the antenna model a characteristic (Fig. 10) of the peak value of the dominant component of the electric field $E(x)$ as a function of the distance from the antenna $x$ has been outlined. The graph also shows the results obtained from the experimental research.

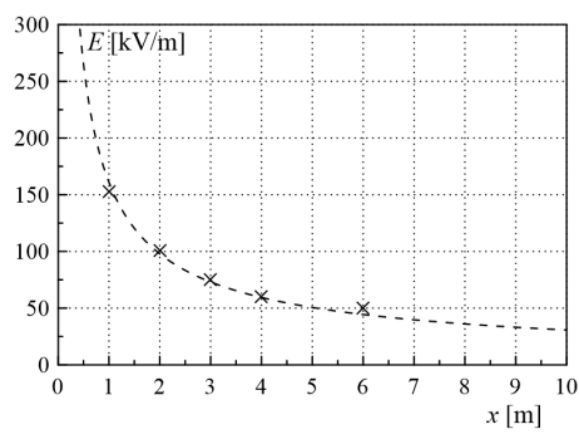

Fig. 6. Characteristics of the peak value of the electrical component $E$ of the electromagnetic field pulse as a function of the distance from the antenna in the axis of the antenna with the measured points.

\section{Conclusions}

Based on the presented simulation and experimental research following conclusions have been drawn:

- Proposed simulation model of the electromagnetic field pulse emission system with high compliance describes the cooperation of the antenna with the other elements of the system as well as its impact on the radiation environment.

- Application of the division of the antenna model into blocks that approximate the individual field zones (reaction and radiation) gives the possibility of analyzing the system in the domain of time and frequency.

- Due to the relatively low operating frequency of the system, it is possible to use it to simulate the emission of electromagnetic field pulses phenomena from natural sources (e.g. atmospheric discharges).

The research has been performed as a part of the project financed by the Polish National Centre for Research and Development - contract DOB-1-1/1/PS/2014.

\section{References}

1. K. Jakubiuk, et. al., ITM Web Conference, vol. 19, 2018 Comp. Appl. in Electrical Engineering (ZKwE'2018).

2. D. Kowalak, et. al., Poznan Univ. of Tech. Acad. Journ., Poznań 2018, vol. 93.

3. M. Nowak, et. al., Poznan Univ. of Tech. Acad. Journ., Poznań 2019, vol. 97.

4. M. Nowak, et. al., Poznan Univ. of Tech. Acad. Journ., Poznań 2018, vol. 93.

5. www.cst.com 